\title{
Misleading questions and the retrieval of the irretrievable
}

\author{
NEAL E. A. KROLL and DEREK A. TIMOURIAN \\ University of California, Davis, California
}

\begin{abstract}
It has been shown that misleading questions between visual presentations and recognition tests lead to incorrect choices, which then appear to completely replace the original memory. In the present experiment, subjects who were "returned to the scene of the crime" after their first recognition test performed markedly better on their second recognition test. This improvement was interpreted as being the result of more efficient retrieval cues provided by the sequential information presented in the return-to-the-scene slides. The model which seemed to best fit the data also assumed that the misleading questions were affecting the guessing bias, rather than memory.
\end{abstract}

In 1980, Loftus and Loftus published an article in the American Psychologist which included an outline of the previous work by E. F. Loftus (e.g., 1975) concerning the apparent memory loss caused by misleading questions asked between the presentation and recognition testing of visual information. Despite a number of attempts on the part of E. F. Loftus and coworkers to elicit retrieval of the previously stored information, this information remained irretrievable, and consequently, Loftus and Loftus concluded that the misleading questions caused information, which had been stored in memory, to be "irrevocably destroyed."

Recently, this conclusion has come under attack from Bekerian and Bowers (1983; Bowers \& Bekerian, 1984), who have published several experiments showing that one obtains the information-loss effect only if the recognition test slides are presented in a different sequence than the original information. If the test sequence is in the same order as the original sequence, then the ordering itself apparently provides enough additional cuing so that the people who had been asked misleading questions do no worse than those who had not received this misleading information.

The Bekerian and Bowers experiments provide evidence against E. F. Loftus's contention (e.g., Cole \& E. F. Loftus, 1979) that memory replacement occurs during the presentation of the conflicting information in the misleading questions. However, there is a possibility that the memory replacement occurs at the time of the recognition test; that is, perhaps the two conflicting sets of information coexist in memory until there is a need to make a decision. At the time of the recognition test, the information from the misleading question, which is more recent-and, because it is being suggested by the experimenter, probably more authoritative-than the information in the original memory trace, causes the person

Send reprint requests to Neal Kroll, Department of Psychology, University of California, Davis, CA 95616. to choose the incorrect slide and then, perhaps, this incorrect slide is remembered as what had been seen.

The current experiment was designed to rule out this possibility by simulating a strategy frequently used to improve retrieval in everyday life; that is, returning to the scene where the events originally occurred. This was achieved in the laboratory by showing slides of the original scene without the characters who had provided the original action. In the main condition of interest, the subjects saw these slides after the first recognition test. Obviously, if memory improves after such order prompting, the memory could not have been "irrevocably destroyed" during either the presentation of the conflicting postevent information or during the initial recognition test.

\section{METHOD}

\section{Subjects}

The data from 432 subjects (144 in each of three conditions) were analyzed. Additional subjects were dropped from the analysis because of equipment failure and occasionally because of an obvious disregard of instructions. The subjects were students in introductory psychology and rhetoric classes who received course credit in exchange for their participation. They were tested in groups of 12 or fewer.

\section{Procedure}

There were three conditions, each of which was divided into six phases. The phases of the main (experimental) condition were:

Phase 1. Subjects saw a sequence of 37 slides which illustrated a walk through a small town and included the observation of a staged purse snatching followed by a chase and capture sequence.

Phase 2. Subjects were given a form with 20 questions about the slides. Three of the questions included information about three critical slides: for any given subject, 2 of these questions gave inconsistent information concerning what was in their critical slides, whereas the remaining question gave consistent information concerning what was in its corresponding critical slide. Questionnaires within a group of 12 subjects were counterbalanced so that three different suggestions were made equally often across subjects. Slides presented across groups of subjects were counterbalanced in such a way that any given piece of information was consistent for one third of the subjects.

Phase 3. The third phase was a 5-min distractor task; subjects were requested to complete a personal information sheet.

Phase 4. Subjects were given the first recognition test. On each of 14 trials, subjects chose one of three slides, which were identical ex- 
cept for one feature. Three of these trials involved the critical slides mentioned above. The presentation of these test trials was in random order and the presentation position of the correct slides was counterbalanced over groups of subjects.

Phase 5. Subjects were "returned to the scene of the crime." This was accomplished by showing them another series of 20 slides that took them through the same town in the same sequence, but at a different time of year and without the characters involved in the original purse snatching. Note that this follows a likely real-life scenario.

Phase 6. After being warned that the questionnaire had included information that may have led them to make errors during the first recognition test, subjects were given a second recognition test.

In addition to the main condition, there were two control conditions. In one, the slides in the return-to-the-scene phase were presented in random order; in the other, these slides were presented in the correct order, but were presented prior to the first recognition test.

\section{RESULTS}

The results are presented in Figure 1. The abscissa represents the percentage of correct choices on the critical trials, with a simple correction for guessing: $C=$ $(3 U-1) / 2$, where $C$ is the corrected percentage and $U$ is the uncorrected percentage correct. The striped bars represent the recognition scores on the original test, and the solid black bars represent the recognition scores on the second test. Each set of four bars represents the scores of the 144 subjects in one of the three conditions, with the first set of four representing the condition of main interest. Within a set of four, the first two bars represent the scores on the critical trials related to consistent information given in the questionnaire, and the second two bars represent the scores on the critical trials related to inconsistent information given in the questionnaire.

Table 1 presents the number of correct and incorrect choices on the critical trials of the second recognition test for the subjects who had previously chosen the slide cor-

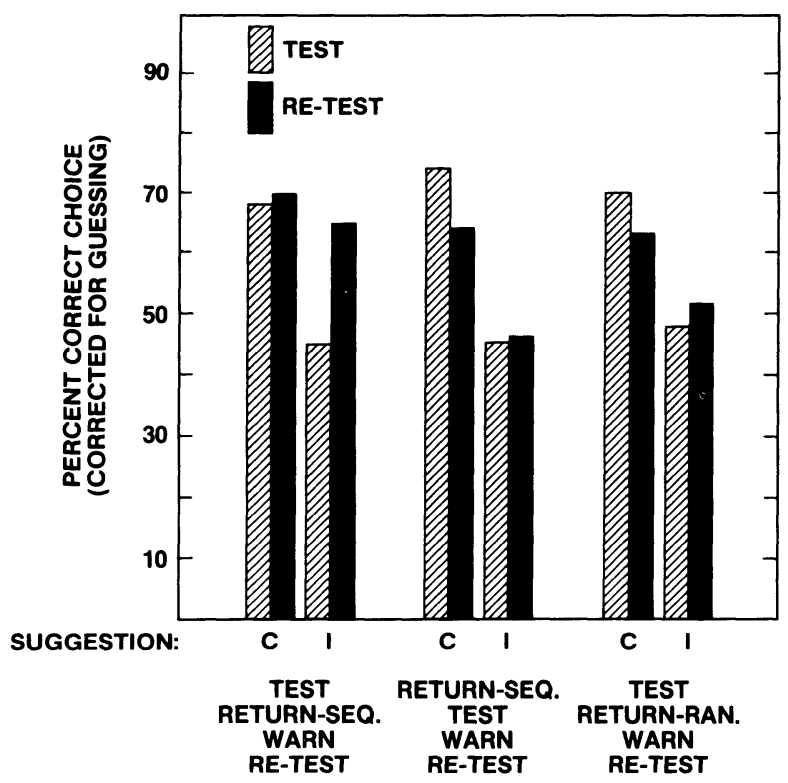

Figure 1. Percentage of correct choices (corrected for guessing) following consistent (C) and inconsistent (I) postevent information presented in the questionnaire for each of the three conditions.
Table 1

Number of Correct and Incorrect Choices on Critical Trials of the Second Test

\begin{tabular}{lcc}
\hline \multicolumn{1}{c}{ Condition } & Correct & Incorrect \\
\hline Test, Sequential Return, Retest & 37 & 33 \\
Sequential Return, Test, Retest & 13 & 63 \\
Test, Random Return, Retest & 19 & 56 \\
\hline
\end{tabular}

responding with the information presented in the misleading question. The subjects who had seen the sequential return-to-the-scene slides after the first test were more likely to choose the correct slide during the second test than were either the subjects who had seen these sequential slides before the first test $\left[\chi^{2}(1)=19.13, p<.01\right]$ or the subjects who had seen a random ordering of these slides after the first test $\left[\chi^{2}(1)=10.44, p<.01\right]$; the subjects in the latter two conditions did not differ reliably from one another $\left[\chi^{2}(1)=1.08, p>.10\right]$.

The lack of any apparent positive effect from seeing the sequential return slides before the first test was a bit of a surprise. It is likely, however, that subjects in this condition were not paying sufficient attention to the return slides since they had not yet experienced the first test and, hence, were not aware of its difficulty.

\section{DISCUSSION}

The results seem to demonstrate both the deleterious effect on memory from the misleading questions and the ability of the sequential order information to provide the additional cuing needed to retrieve the correct information masked by the conflicting information presented within the misleading questions. The improvement in the scores after viewing the sequential return-to-the scene slides should not occur according to the hypothesis that the information is destroyed during the presentation of inconsistent information. Nor should it occur if the information is destroyed during the first recognition test-a possibility allowed by Bekerian and Bowers's (1983; Bowers \& Bekerian, 1984) experiments. Obviously, if the information had actually been destroyed at any time, it could not be retrieved. Thus, these results suggest that the information is not destroyed, but is simply difficult to retrieve, and that the sequentially presented return-to-the-scene slides provide particularly good retrieval cues.

Bekerian and Bowers are not alone in challenging Loftus and Loftus's (1980) interpretation of the results of the misleading question experiments. McCloskey and Zaragoza (1985a, 1985b) noted that the information provided in the postevent questionnaire should bias the guessing rates in such a way that, in terms of Figure 1, correcting for guessing in the ordinary way should tend to undercorrect on items for which the postevent information is consistent (subjects who do not remember the original event are assumed to be biased toward the correct answer by the postevent questionnaire), and it should overcorrect on items for which the postevent information is inconsistent (subjects who do not-and perhaps even some who do-remember the event are assumed to be biased toward the incorrect answer by the misleading questions). On this basis, one can explain the E. F. Loftus (e.g., Loftus \& Loftus, 1980 results without hypothesizing any memory distortions. The results of the Bekerian-Bowers experiments are incompatible with this analysis: the memory of the misled subjects with the recognition test in sequence is too good. That is, when the McCloskey and Zaragoza analysis of the guessing bias is applied to the Bekerian-Bowers experiments, the true memory for the items paired with inconsistent information appears to be better than for the items paired with consistent information.

McCloskey and Zaragoza (1985a) attempted to replicate the BekerianBowers results, but did not get their effect. McCloskey and Zaragoza used many more subjects than Bekerian and Bowers had in their first experiment and, thus, their results should be more reliable. There are 
two counterarguments to this: (1) they compared their results to only one of the Bekerian-Bowers experiments, but there have been several replications (Bowers \& Bekerian, 1984; Bekerian, personal communication); and (2) they give no details of their experiment, so it is difficult to be certain that they did not change some critical part of the BekerianBowers procedures. On the other hand, McCloskey and Zaragoza (personal communication) have assured us that they have attempted several replications and have tried to make their procedures as similar as possible to those of Bekerian and Bowers.

The results of the present experiment seem to provide a similar contradiction to the McCloskey-Zaragoza analysis. To illustrate this, Figure 2a depicts our results corrected for the guessing hypothesis of McCloskey and Zaragoza. The first two bars represent the corrected test and retest scores averaged over all critical trials following consistent information. The second two bars represent the corrected test and retest scores on the critical trials following inconsistent information for the combined subjects in the two control conditions. The fact that these two bars are identical to the first two is no accident: the correction procedure defined the guessing-bias factor by finding what was needed to make them identical. For simplification, positive and negative biases, presented in Figure $2 b$, were assumed to be equal. Adding the bias factor to $33.3 \%$ gives the assumed guessing percentage for the critical trials paired with consistent information; subtracting one half of the bias factor from $33.3 \%$ gives the assumed guessing percentage for the critical trials paired with inconsistent information. According to this interpretation of the results, the control subjects showed a smaller biasing effect from the misleading questions on the retest than they had originally, but there was virtually no forgetting of the information in original slides. When the same retest bias factor is applied to the critical trials paired with inconsistent information in the experimental condition, the result (Bar 6 in Figure 2a) is incompatible with the assumptions: These subjects seem to exhibit a large, unexplainable reminiscence effect.

How can this be resolved? One possibility is that the assumptions of McCloskey and Zaragoza (1985a) are incorrect. E. F. Loftus, Miller, and Burns (1978) found no advantage of the consistent information in the questionnaire over no relevant information in the questionnaire, when the questionnaire was administered shortly after the event and when the retention interval was short. This finding, taken together with the Bekerian-Bowers results and the results of the present experiment, may be an indication that the information in the questionnaire affects the guessing rate only slightly, if at all. The postevent information, after all, is typically not a salient feature of the question in which it is embedded. Thus, perhaps, it is only incorporated into a person's memory if it relates to something already stored, as long as it is not salient enough to.

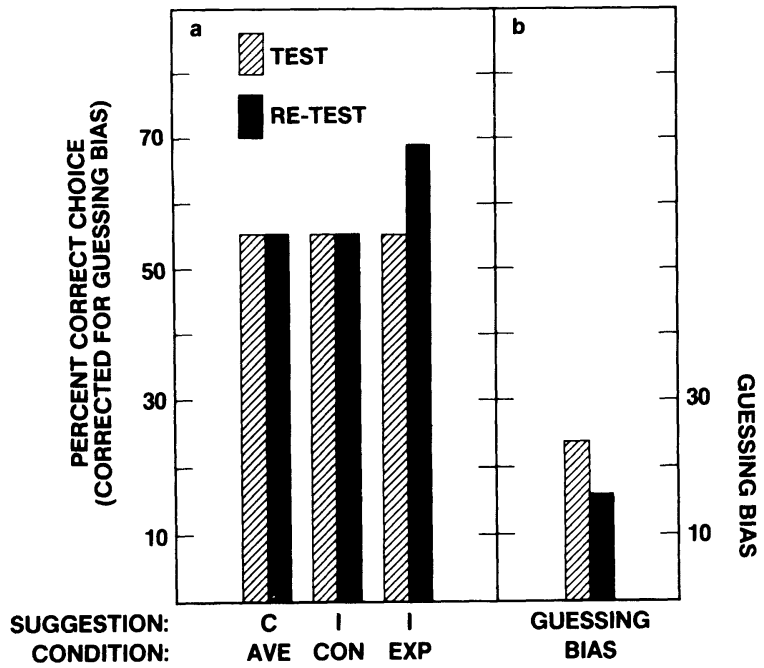

Figure 2. Percentage of correct choices (corrected for guessing with the guessing bias shown) for the consistent (C) trials averaged over all conditions (AVE), and for the inconsistent (I) trials separated by control (CON) and experimental (EXP) conditions.

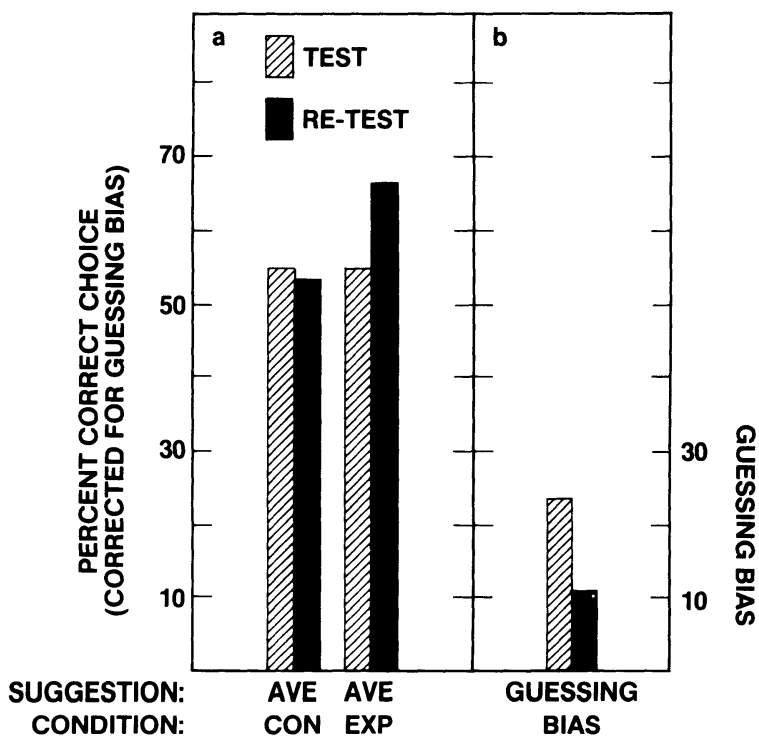

Figure 3. Percentage of correct choices (corrected for guessing with the guessing bias shown) under the assumption of an overall retrieval improvement due to the sequential return slides' being shown between tests.

cause the subject to question an inconsistency. Opposed to this explanation, however, are the findings that misleading questions apparently have the power to lead subjects to recall and recognize features that they have never seen (Loftus, 1975; Loftus \& Greene, 1980).

Another possible explanation of the present results was suggested to us by McCloskey (personal communication). Perhaps the return slides result in a general improvement in memory, quite independent from any effect of the intervening questions. This might be seen as an example of the more general phenomenon of improving memory through the reinstatement of the original context (e.g., Davies \& Milne, 1985). In support of this explanation, our experimental group shows not only a large improvement on the inconsistent trials, but also a small improvement on the consistent trials (see Figure 1).

Figure 3 a depicts the current data corrected with this added assumption in mind. The first two bars represent the assumed true scores of the two control conditions on the critical trials of the test and retest, respectively. The bars in Figure $3 \mathrm{~b}$ represent the guessing biases on the two tests. We are again assuming the simple case where the positive and negative biases are equal. Consequently, adding the guessing bias to $33.3 \%$ gives the assumed guessing rate for consistent trials, and subtracting one half of the guessing bias from $33.3 \%$ gives the assumed guessing rate for inconsistent trials. Note that, with these assumptions, there is an indication that, as one moves from the test to the retest, there is both a small drop in memory for the initial slides and a larger drop in biasing from the intervening questions.

Bars 3 and 4 of Figure $3 a$ represent the assumed true scores of the experimental conditions; the guessing biases are assumed to be the same as for the control conditions. This seems to give a reasonable fit to the current data. The major assumptions are: (1) that misleading questions do not really affect memory-only the guessing biases-and (2) that sequential return-to-the-scene slides inserted between the two recognition tests provide impressive retrieval cues. However, the parameters used to fit the model are completely post hoc. What is needed now is a replication with additional conditions which allow for independent estimations of the parameters.

\section{REFERENCES}

Bekerian, D. A., \& Bowers, J. M. (1983). Eyewitness testimony: Were we misled? Journal of Experimental Psychology: Learning, Memory, \& Cognition, 9, 139-145. 
Bowers, J. M., \& BeKerian, D. A. (1984). When will post-event information distort eyewitness testimony? Journal of Applied Psychology, 69, 466-472.

Cole, W. G., \& LofTUS, E. F. (1979). Incorporating new information into memory. American Journal of Psychology, 92, 413-425.

Davies, G., \& Milne, A. (1985). Eyewitness composite producton: A function of mental or physical reinstatement of context. Criminal Justice \& Behavior, 12, 209-220.

LoFTUs, E. F. (1975). Leading questions and the eyewitness report. Cognitive Psychology, 7, 560-572.

LoFTUS, E. F., \& GREENE, E. (1980). Warning: Even memory for faces may be contagious. Law \& Human Behavior, 4, 323-334.

LofTUS, E. F., \& LoFTUS, G. R. (1980). On the permanence of stored information in the human brain. American Psychologist, 35, 409-420.
Loftus, E. F., Miller, D. G., \& BuRNS, H. J. (1978). Semantic intergration of verbal information into visual memory. Journal of Experimental Pschology: Human Learning \& Memory, 4, 19-31.

MCCloskeY, M. , \& ZARAGozA, M. (1985a). Misleading postevent information and memory for events: Arguments and evidence against memory impairment hypotheses. Journal of Experimental Psychology: General, 114, 1-16.

McCloskey, M., \& ZaragozA, M. (1985b). Postevent information and memory: Reply to Loftus, Schooler, \& Wagenaar. Journal of Experimental Psychology: General, 114, 381-387.

(Manuscript received for publication December 16, 1985.) 